\title{
Génétique moléculaire du cancer du col de l'utérus
}

Le cancer du col de l'utérus continue de poser un réel problème de santé, même si dans les pays développés sa fréquence décroît grâce au dépistage précoce des lésions précancéreuses. Les cellules cancéreuses du col sont presque toujours associées à la présence dans leur génome de l'ADN de papillomavirus de type 16 ou 18. Il existe, de plus, des anomalies des oncogènes dont la plus fréquente semble être l'hyper-expression de $c-m y c$, associée ou non à une amplification génique. L'hyper-expression de $c$-myc semble à ce jour constituer l'un des principaux éléments pronostique des cancers du col, et pourrait donc être d'un grand intérêt dans l'établissement du protocole thérapeutique chez les malades atteintes de ces cancers.

\section{Guy Riou}

Professeur de biochimie et de biologie moléculaire.

Directeur de laboratoire

\section{RÉFÉRENCES}

1. Parkin DM, Stjernsward J, Muir CS. Estimates of the worldwide frequency of twelve major cancers. Bull WHO 1984;62:163-82.

2. Hall SW, Monaghan JM. Invasive carcinoma of the cervix in younger women. Lancet 1983 ; ii : 731 .

3. Van Bommel PFJ, Van Lindert ACM, Kock HCLV, Leers WH, Neijt JP. A review of prognostic factors in early-stage carcinoma of the cervix (FIGO I B and II A) and implications for treatment strategy. Eur J Obstet Gynecol Reprod Biol 1987 ; 26 : 69-84.

\section{ADRESSE ET TIRÉS A PART}

G. Riou : laboratoire de pharmacologie clinique et moléculaire, Institut Gustave-Roussy, 94800 Villejuif, France.

e cancer du col de l'utérus est le deuxième cancer de la femme dans le monde [1]. Dans de nombreux pays de l'Amérique du Sud et de l'Afrique, il représente même la première cause de mortalité par cancer et sa fréquence ne cesse de croître. En revanche, dans la majorité des pays développés, le cancer du col de l'utérus est moins fréquent et accuse même une nette régression attribuée pour une part aux efforts intenses de dépistage précoce. Cependant on constate une augmentation du nombre des cancers chez la femme de moins de 35 ans, et cette situation est d'autant plus préoccupante que les cancers de ces femmes jeunes semblent plus agressifs quel que soit le stade clinique auquel il est dépisté [2]. D'une manière générale, quand le cancer est diagnostiqué à des stades précoces, environ 15 à $20 \%$ des malades rechutent après leur traitement. Bien souvent les rechutes échappent à toute prévision, ce qui souligne la complexité du comporte- ment biologique de la cellule cancéreuse. Plusieurs facteurs pronostiques des cancers du col de stades précoces sont connus mais ne sont pas toujours fiables [3]. Ils ne permettent pas de mettre en œuvre une thérapeutique qui pourrait alors se révéler plus efficace. Les récentes découvertes dans le domaine des oncogènes et l'application des techniques de biologie moléculaire à la clinique ont donné un nouvel essor à la recherche de nouveaux marqueurs biologiques.

\section{Épidémiologie}

De nombreuses études épidémiologiques ont suggéré depuis longtemps qu'un agent infectieux transmis par voie vénérienne était impliqué dans la genèse des cancers du col de l'utérus $[4,5]$. En effet, ces études ont permis de montrer qu'il existait une association étroite entre le développement du cancer et le comportement sexuel des malades et de leurs partenaires mâles. Le risque de déve- 


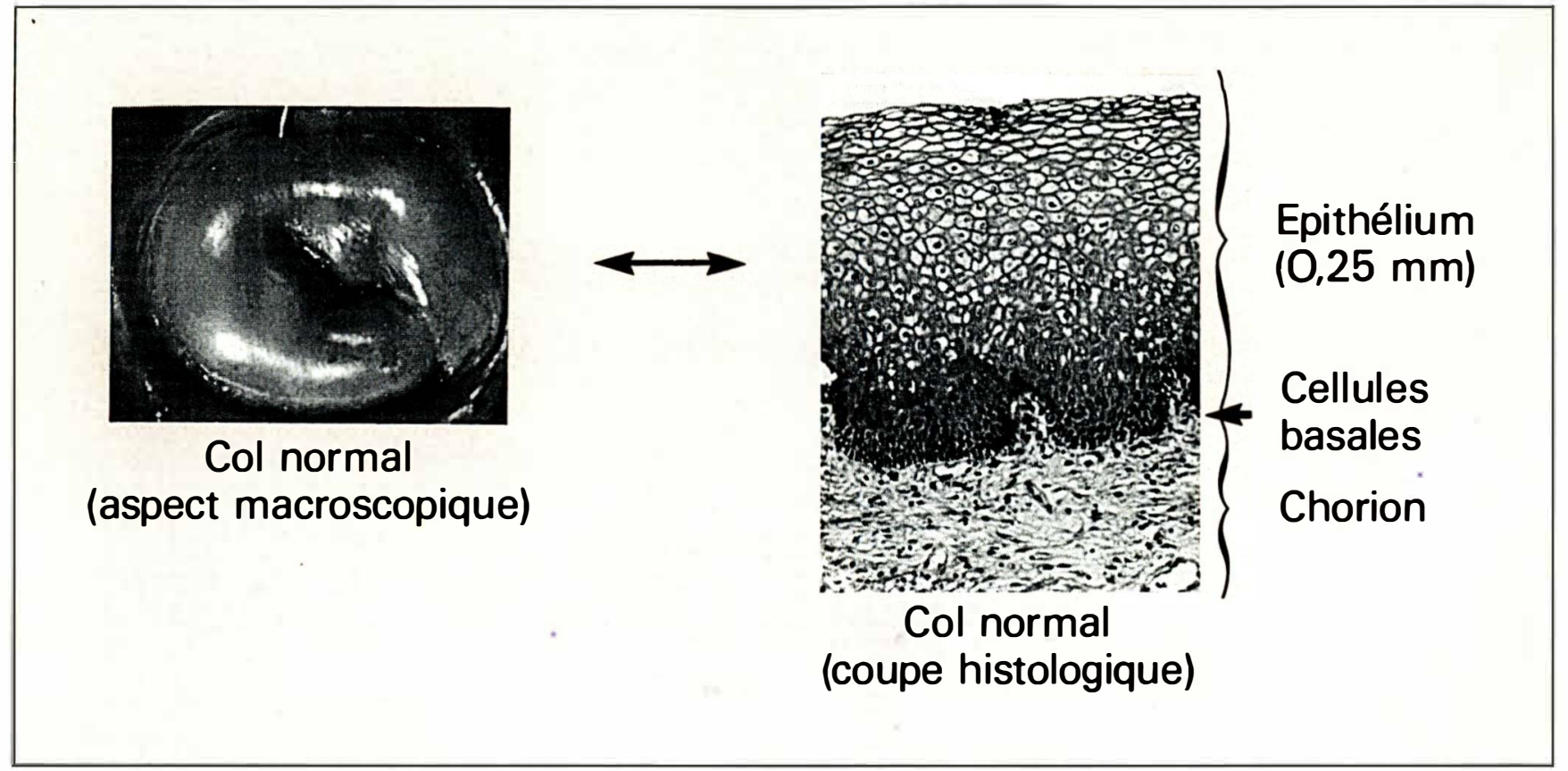

Figure 1. Col de l'utérus normal.

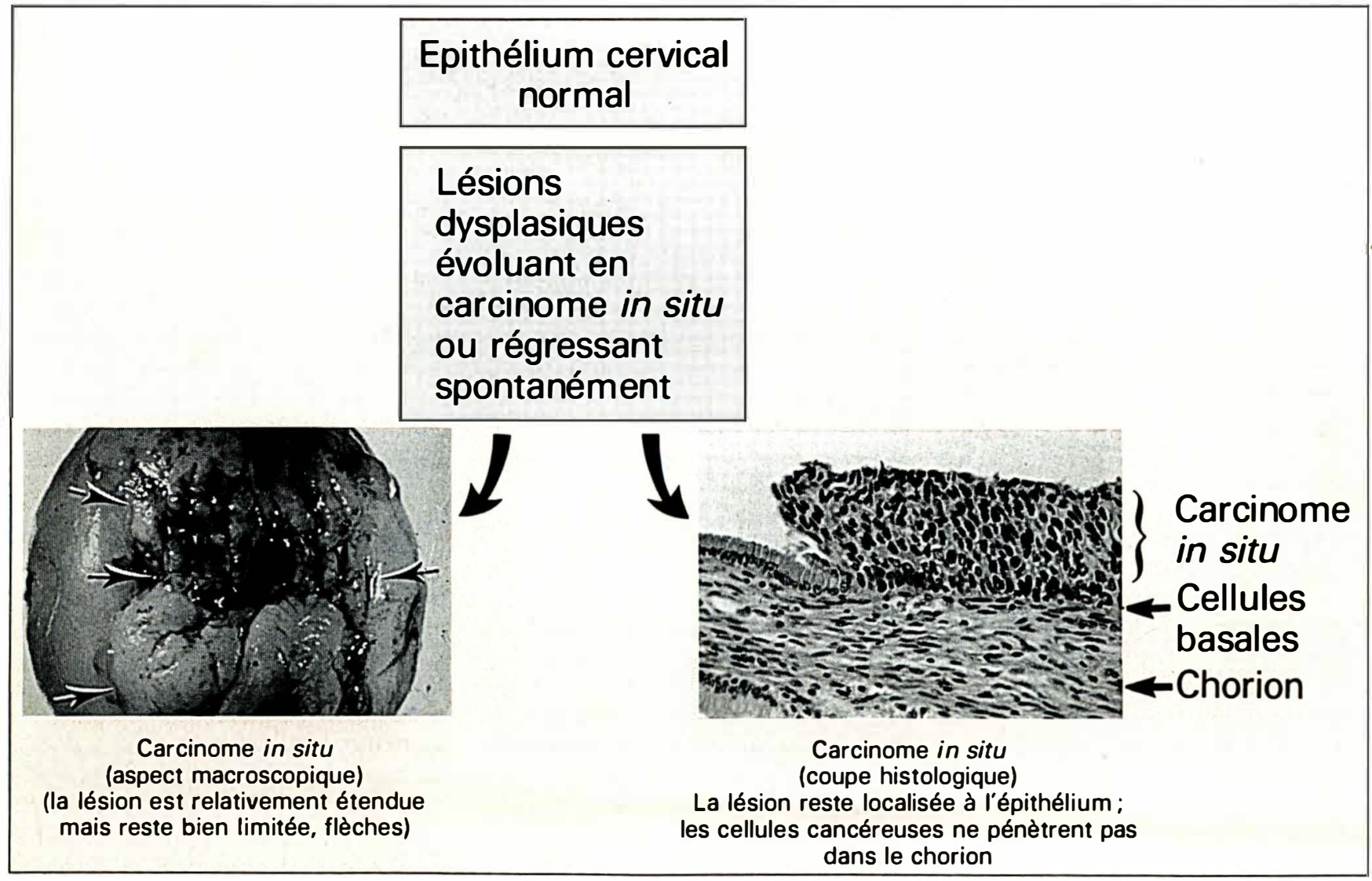




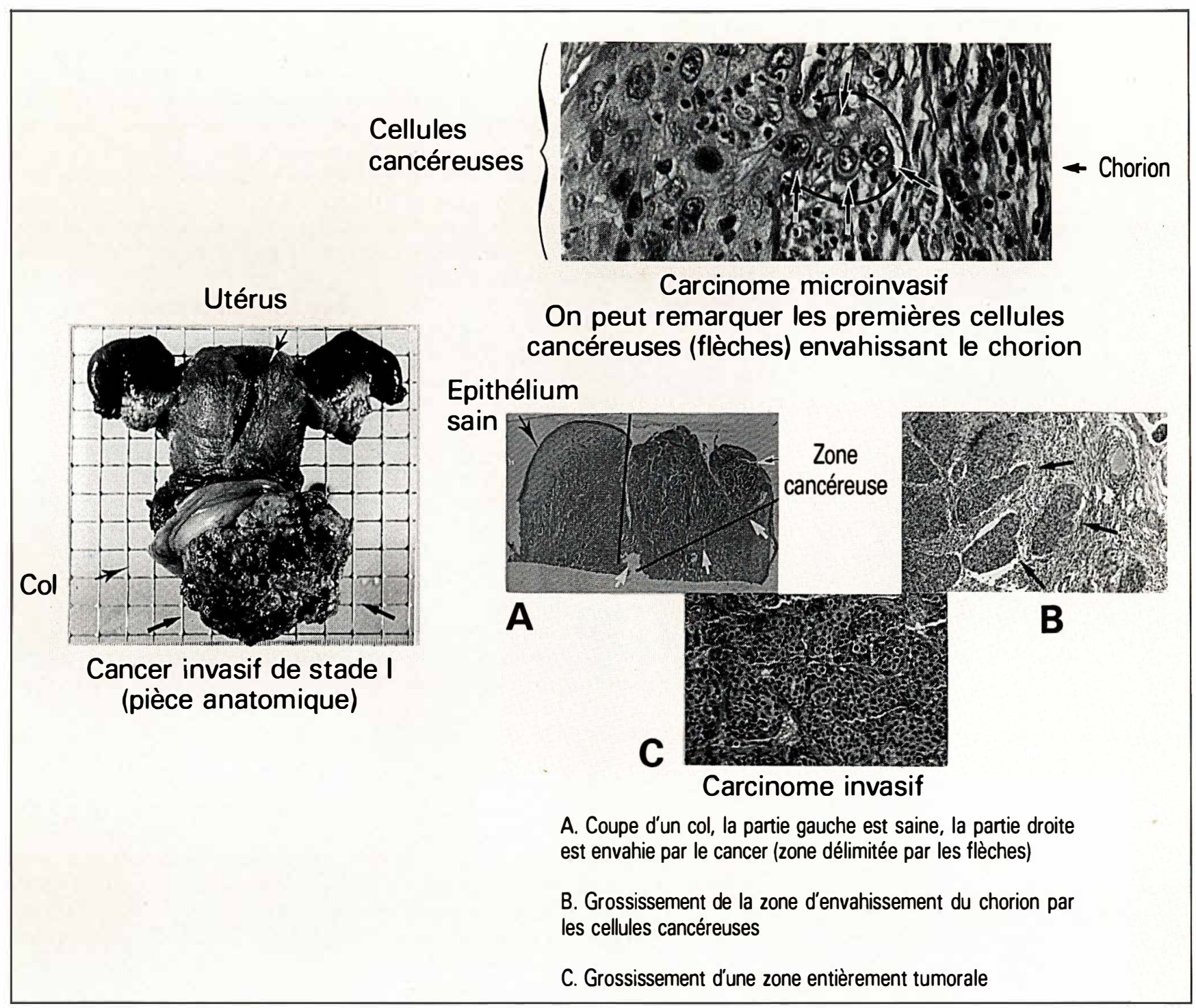

Figure 3. Col de l'utérus présentant un cancer invasif.

lopper un cancer du col est très significativement augmenté lorsque les premiers rapports sexuels soṇt précoces et poursuivis avec de nombreux partenaires. D'autres facteurs liés à des mauvaises conditions de vie et à une hygiène médiocre favorisent également le cancer du col. Plusieurs études séro-épidémiologiques ont suggéré que l'herpétovirus de type 2 pouvait être l'un de ces agents. Cependant le rôle de ce virus est maintenant très controversé. Une étude prospective importante dénie même tout rôle à l'herpétovirus dans l'étiologie des cancers du col [6].

Les papillomavirus humains (PVH) ont été suspectés, mais il a fallu attendre l'application des techniques nouvelles de génétique moléculaire pour isoler de nouveaux types viraux $\mathrm{m} / \mathrm{s} n^{\circ} 7 \mathrm{vol} .4$, seplembre 88 dans les cancers du col. Nous développerons ces résultats dans un chapitre ultérieur.

\section{Histoire naturelle des cancers du col de l'utérus}

Plus de $85 \%$ des cancers du col sont d'origine épidermoïde. Le revêtement cellulaire externe du col de l'utérus est constitué de couches de cellules épithéliales formant une épaisseur d'environ $0,25 \mathrm{~mm}$. Seules les cellules de la zone interne formée de cellules basales possèdent la propriété de se diviser et engendrent les strates de cellules kératinisées (figure 1). La transformation d'une cellule normale en une cellule cancéreuse met en œuvre un processus complexe comportant de nom-

breuses étapes au cours desquelles des lésions précancéreuses (dysplasies) précèdent les lésions de carcinome in situ (figure 2) et les cancers invasifs (figure 3). Le diagnostic clinico-pathologique de ces lésions est important car leur éradication empêche leur développement ultérieur en cancer invasif. Dans la plupart des cas, les lésions de dysplasie régressent spontanément, parfois elles évoluent en carcinome in situ puis invasif. Il est possible de déceler le tout début de l'invasion (carcinome micro-invasif, figure 3 ).

Lorsque le diagnostic d'un cancer invasif a été établi, il est de toute évidence primordial de déterminer avec la plus grande précision le stade clinique, puisque c'est de cet examen que dépend le traitement. D'une 
manière générale les gynécologues se réfèrent à la classification FIGO établie par la fédération internationale de gynécologie et d'obstétrique avec quelques variations propres à la fédération des centres anti-cancéreux. Nous avons reproduit d'une manière très schématique l'envahissement tumoral correspondant aux quatre stades cliniques (figure 4). Les chances de survie à cinq ans sont faibles pour les patientes ayant un cancer de stade III ou IV [7]. En revanche, seulement 15 à $20 \%$ des patientes atteintes d'un cancer de stades I ou IIp vont mourir de leur cancer [3]. D'autres facteurs pronostiques des cancers des stades précoces (I et II) sont déterminés selon plusieurs critères clinico-biologiques. Comme dans la majorité des cancers, l'envahissement ganglionnaire est le facteur pronostique le plus fiable [3, 8]. Le nombre de ganglions tumoraux et leur localisation renseignent le clinicien sur les risques de rechute. Lorsque les ganglions de la sphère lombo-aortique sont envahis les risques de diffusion métastatique sont élevés. La taille de la tumeur semble également importante, une tumeur de grande taille a une plus grande faculté de disséminer ses cellules que les tumeurs de petite taille. L'origine histologique joue de même un certain rôle, les adénocarcinomes qui représentent environ $10 \%$ des cancers du col étant plus agressifs que les cancers d'origine épidermoïde. Il existe très peu d'analyses multifactorielles prenant en compte toutes ces données pronostiques, ce qui rend difficile la détermination de l'importance relative de chacun de ces facteurs, d'autant plus que ceux-ci sont souvent liés. En principe, les méthodes de traitement des cancers du col de stade précoce découlent des données pronostiques et ont donc une incidence directe sur les rechutes du cancer. Aussi était-il important de mettre à la disposition des cliniciens de nouveaux marqueurs biologiques qui, associés à certains facteurs clinico-pathologiques, permettraient de prédire avec plus de précision le risque de récidive. Nous nous proposons de résumer succintement les travaux suggérant le rôle joué par les papillomavirus humains dans le développement et la progression des cancers du col de l'utérus et l'impor- tance de leur détection dans les lésions précancéreuses. Nous résumerons également les résultats des premiers travaux qui indiquent que certains proto-oncogènes amplifiés et/ou surexprimés sont associés à la progression des cancers du col et que, détectés dans les cancers de stade précoce, ils semblent avoir une valeur pronostique non négligeable.

\section{Les papillomavirus}

Plus de 50 types distincts de papillomavirus humains (PVH) différant, par leur génotype ont été actuellement décrits et ce nombre ne cesse de croître. Chaque type de PVH est souvent associé à une symptomatologie spécifique, principalement de la peau et des voies ano-génitales. Des travaux datant déjà de plus de dix ans avaient montré, dans les lésions précancéreuses du col, la présence de PVH dans des cellules présentant une morphologie très typique, les koïlocytes. Plus tard, grâce aux progrès de la biologie moléculaire divers types de PVH ont été isolés de lésions bénignes d'origine génitale. Leurs séquences virales ont servi par la suite à cloner d'autres types viraux. Par définition, on considère qu'un PVH représente un nouveau type si son $\mathrm{ADN}$ génomique possède moins de $50 \%$ d'homologie avec l'ADN des autres types définis. C'est ainsi que le groupe de Zur Hausen [9, 10] a réussi à cloner directement à partir de cancers du col, deux nouveaux types, le PVHI6 et le PVH18. L'infection du col par ces PVH semble se produire à la faveur d'une microlésion exposant les cellules de la couche basale au virus. Les lésions infectées sont caractérisées par des atypies nucléaires marquées, des figures mitotiques anormales, un caryotype aneuploïde. Ce sont ces lésions qui seraient les précurseurs des cancers invasifs [11]. Zur Hausen et al. $[9,10]$ ont mis en évidence les PVHl6 et PVH 18 dans plus de $70 \%$ des cancers invasifs du col avec une forte prévalence du PVHl6. En collaboration avec G. Orth (Institut Pasteur, Paris), nous avons confirmé ces résultats [12, 13]. Dans une étude récente $(\mathrm{G}$. Riou et $\mathrm{G}$. Orth en préparation) portant sur plus de 150 cancers invasifs du col de type épidermoïde et de diffé- 
rents stades cliniques, nous avons montré que: (1) les PVH sont présents dans plus de $90 \%$ des tumeurs quel que soit le stade clinique: (2) les PVH des types 16 et 18 sont de loin les plus fréquents, ils représentent respectivement 65 et $15 \%$ des tumeurs positives pour les PHV. D'autres types isolés récemment, les PVH33, 35 et $39[14,15]$ ont été également détectés. Environ $5 \%$ des cancers contiennent un second type viral en plus du PVHl6. La présence de deux virus dans ces cancers ne semble pas leur conférer une agressivité particulière; (3) les séquences virales sont dans leur grande majorité réarrangées et intégrées dans le génome cellulaire. On observe une à plusieurs centaines de copies virales. Le nombre de copies et le type du virus ne semblent pas associés à la progres- sion tumorale; 4) des transcrits viraux ont été détectés dans plus de $80 \%$ des cancers. Leur nombre et leur taille sont variables.

Quelles conclusions peut-on tirer de ces résultats et du rôle du PVH dans le développement et la progression des cancers invasifs du col? Le rôle du PVH dans l'initiation du cancer du col ne semble plus faire de doute, mais dans la plupart des cas le temps de latence entre l'infection d'une cellule du col par un PVH et la transformation en lésions cancéreuses est long. Des résultats récents [16], obtenus à partir de prélèvements par frottis du col utérin dans une population de femmes venant consulter sans trouble apparent, viennent de montrer la présence des PVHl6 ou 18 dans plus de $5 \%$ des cas. Ces séquences virales ont été détectées

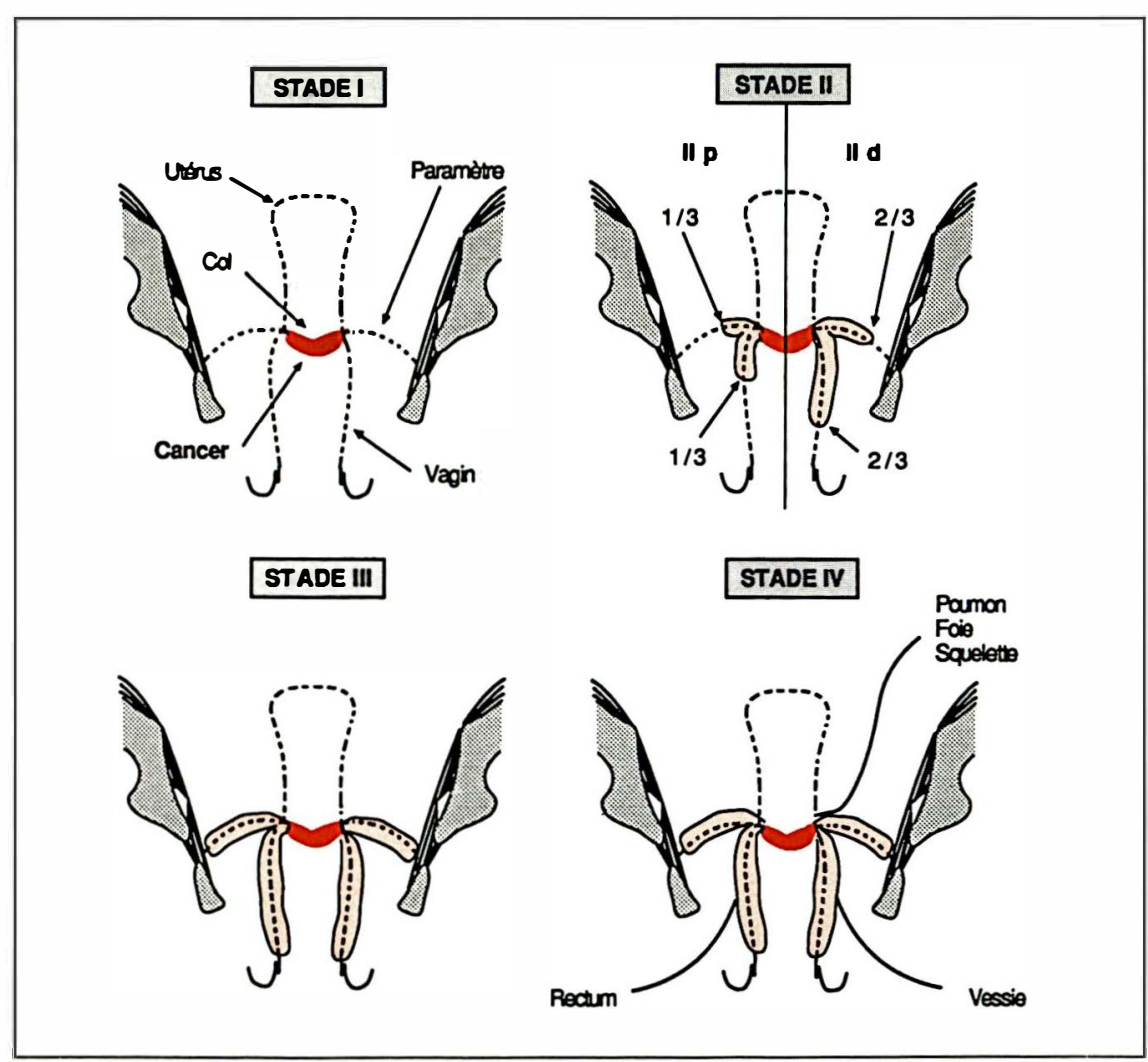

Figure 4. Représentation schématique de l'envahissement tumoral du col de l'utérus au cours des quatre stades cliniques. Stade I: le cancer reste localisé au col. Stade II: le cancer envahit le vagin et/ou les paramètres unilatéralement ou bilatéralement. L'extension de l'envahissement définit les stades IIp et IId. Stade III: le vagin et/ou les paramètres sont envahis. Stade IV: le cancer envahit les organes annexes (vessie, rectum) ou forme des métastases à distance (foie, poumon, squelette osseux). surtout dans des dysplasies sévères ou des carcinomes in situ (64\%). Cette étude ainsi que d'autres [17] sugge'rent que la présence de ces virus constitue pour les malades un risque de développer des néoplasies cervicales intra-épithéliales ou invasives. Sur le plan étiologique, il est intéressant de rappeler l'importance du rôle du partenaire sexuel mâle dans la transmission de l'infection à PVH [18]. Le PVH est présent et transcrit aussi bien dans les tumeurs des stades précoces que dans celles des stades évolués ainsi que dans les métastases. Ceci suggère que le PVH pourrait être utile au maintien de l'état malin. Cependant, il apparaît tout à fait certain que d'autres facteurs sont nécessaires pour effectuer la transformation maligne. Les questions restent encore sans réponse quant au rôle que pourraient jouer les $\mathrm{PVH}$ dans l'activation de gènes en particulier des proto-encogènes, bien qu'une étude récente faite sur certaines lignées de cancer du col utérin suggère que le $\mathrm{PVH}$ pourrait provoquer une activation en $c i s^{*}$ de l'oncogène $c$-myc [19]

\section{Les oncogènes cellulaires}

Il ne fait plus de doute que les cancers humains ont pour origine un mauvais fonctionnement de certains gènes, surtout depuis la découverte dans les tumeurs, de proto-oncogènes dont la structure et (ou) le fonctionnement sont altérés.

Les proto-oncogènes font partie du patrimoine génétique des cellules normales de toutes les espèces de vertébrés qui ont été analysées. Ils ont été conservés au cours de l'évolution, ce qui suppose un rôle important pour l'économie de la cellule. Bien que leur fonction biologique soit souvent méconnue, ils semblent cependant directement impliqués dans les processus de croissance et de différenciation cellulaire. Ils sont capables d'induire la transformation maligne après certaine modification de leur structure ou de leur fonctionnement : réarrangement du gène avec ou sans translocation chromosomique, amplifica-

* Activation en cis: due à un effet de proximité sur le brin d'ADN. 
tion, mutation, surexpression [2022].

Ces anomalies ont été tout d'abord décelées dans des lignées cancéreuses puis dans des cancers humains de différents types. Nous allons résumer les travaux effectués dans notre laboratoire qui montrent le rôle des oncogènes $c$-myc et $c$-Ha-ras dans la progression des cancers du col. Nous montrerons également l'application possible de la recherche de leur activation dans l'établissement du pronostic.

- L'amplification et la surexpression de l'oncogène $c$-myc sont associées à la progression tumorale. La structure de l'oncogène $c-m y c$ a été caractérisée dans 128 échantillons de cancers épidermoïdes du col à différents stades cliniques. Une amplification du gène a été décelée dans environ $25 \%$ des cancers sans aucune évidence d'un réarrangement du gène (figure 5). La transcription de ce gène $a$ été analysée par hybrida- tion moléculaire sur réplique (Northern blot et slot blot). Le transcrit $c-m y c$ de $2,4 \mathrm{~kb}^{*}$ généralement observé dans les tissus humains normaux, a été décelé dans les 106 échantillons de col étudiés. Des taux élevés de transcrits ont été obtenus dans environ $48 \%$ de ces cancers. La surexpression de l'oncogène $c-m y c$ ne dépend pas de l'état physique du gène puisqu'elle peut être observée quand le gène est amplifié ou présent à l'état d'une copie unique (figure 5). Les résultats montrent que l'activation de l'oncogène $c$-myc (amplification ou surexpression) est plus fréquente dans les cancers des stades évolués que dans ceux des stades précoces (Tableau I). Les altérations du gène $c-m y c$ sont significativement associées à la progression des cancers du col de l'utérus [12, 13, 23].

${ }^{*} k b=$ kilobases.
- La surexpression de $c-m y c$ a une valeur pronostique dans les cancers de stade précoce. Il nous a semblé intéressant de suivre l'évolution clinique des malades ayant un cancer de stade précoce en fonction de l'expression de $c-m y c$. Nous avons inclus 72 malades dans notre étude. La distribution de la surexpression de $c$ $m y c$ a été évaluée en fonction de 4 facteurs pronostiques potentiels (envahissement ganglionnaire, âge, stade clinique, et origine géographique des malades). Nous avons ensuite analysé le risque relatif de rechute des malades en fonction de ces 4 facteurs et de la surexpression du gène $c-m y c$ (Tableau II). Les résultats de l'analyse unifactorielle montrent que le risque de rechute est associé à la surexpression de $c-m y c$, à l'envahissement ganglionnaire et à l'origine géographique des patientes. Les résultats de l'analyse multifactorielle montrent que le risque de rechute des malades est associé uni-

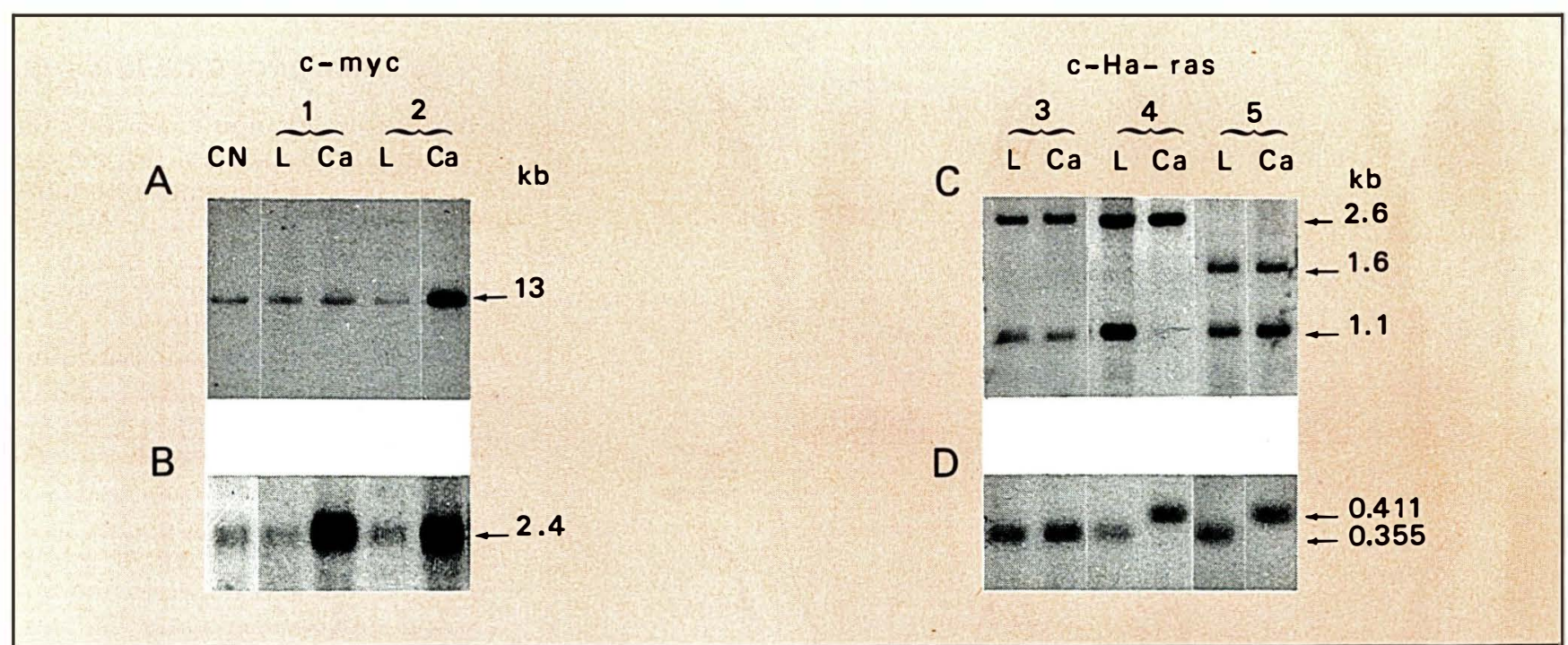

Figure 5. Analyse des gènes c-myc et c-Ha-ras dans les cancers du col de l'utérus. A : L'ADN préparé à partir des cancers $(\mathrm{Ca})$ et des lymphocytes $(L)$ des malades nos 1 et 2 a été digéré par l'enzyme de restriction EcoRl et analysé par hybridation moléculaire sur réplique (Southern) avec une sonde du gène c-myc humain ( $3^{\ominus}$ exon). Après autoradiographie on révèle un gène amplifié dans le cancer $(\mathrm{Ca})$ du malade $n^{\circ} 2$. B : L'ARN préparé à partir des échantillons précédents a été analysé par hybridation moléculaire sur réplique (Northern) avec la sonde c-myc. Après autoradiographie on révèle des niveaux élevés de transcrits dans les cancers des malades nos 1 et 2. C et D: L'ADN préparé à partir de lymphocytes (L) et de cancers (Ca) des malades nos 3, 4 et 5 a été digéré par l'enzyme de restriction Mspl et analysé par hybridation moléculaire sur réplique (Southern). C : I'hybridation est faite avec une sonde c-Ha-ras-VTR pour mettre en évidence le polymorphisme génétique. Après autoradiographie, on décèle la perte de l'allèle Ha-ras de 1,1 kb dans le cancer de la malade no4. D: I'hybridation est faite avec la sonde Ha-ras (fragment de 0,6 kb Xmal-Xmal) pour révéler la présence sur le codon 12 d'une mutation qui supprime le site de restriction de Mspl. Après autoradiographie, on décèle un fragment de 411 paires de base dans les cancers des malades nos 4 et 5 signifiant que le gène c-Ha-ras est affecté d'une mutation. Dans les lymphocytes des malades et le cancer de la malade $n^{\circ} 3$, on révèle deux fragments d'ADN de 355 et 56 paires de base montrant que le gène ne porte pas de mutation. Le fragment d'ADN de 56 paires de base n'est pas visible dans nos conditions expérimentales. 
Tableau I

AMPLIFICATION ET SUREXPRESSION DU GĖNE C-MYC DANS LES CANCERS ÉPIDERMOIDES DU COL DE L'UTÉRUS DES STADES CLINIQUES PRÉCOCES (STADES I ET II) ET ÉVOLUÉS (STADES III ET IV)

\begin{tabular}{|c|c|c|}
\hline Stades cliniques & \multicolumn{2}{|c|}{$\begin{array}{c}\text { Nombre de tumeurs ayant une altération } \\
\text { de myc/Nombre de tumeurs examinées } \\
\text { Amplification } \\
\text { Surexpression }\end{array}$} \\
\hline I + II & $\begin{array}{c}9 / 83 \\
(10,8 \%)\end{array}$ & $\begin{array}{c}25 / 74 \\
(33,8 \%) \\
23 / 45\left(p<10^{-4}\right)\end{array}$ \\
$21 / 27\left(p<10^{-3}\right)$ \\
$(51,1 \%)$
\end{tabular}

quement à la surexpression de $c-m y c$ et à l'envahissement ganglionnaire (Tableau II). La surexpression de $c$ myc semble même être un facteur pronostique plus puissant que l'envahissement ganglionnaire [23].

Par ailleurs, l'étude de la survie sans rechute des malades pendant 18 mois montre que $51 \%$ des malades présentant une surexpression de $c$-myc dans leur tumeur font une rechute, alors que seulement $10 \%$ des malades de l'autre catégorie font une rechute (figure 6). Cette étude doit être poursuivie sur une période de temps plus longue pour savoir si ces résultats sont encore acquis à moyen et long terme. Néanmoins, la surexpression de $c-m y c$ dans les tumeurs de stade précoce semble avoir une valeur pronostique très forte à court terme.

- La distribution des allèles $c-H a$ ras dans les cancers du col de l'utérus ne diffère pas de celle d'une population non affectée. Des études relativement récentes ont montré que le locus c-Ha-ras présentait un polymorphisme vis-à-vis de plusieurs enzymes de restriction dont MspI. Deux sites de restriction encadrent une région située en 3' du 4 $4^{\mathrm{e}}$ exon du gène $c$-Ha-ras. Cette région est constituée par une séquence répétée en tandem de 28 paires de bases (variable tandem repetition, VTR) dont le nombre peut varier d'un allèle à l'autre. Lorsque l'ADN génomique est clivé par MspI puis analysé par hybridation moléculaire sur réplique avec le gène $c$-Ha-ras VTR on révèle une ou deux bandes d'ADN chez les individus homozygotes et hétérozygotes respectivement. Une $\mathrm{m} / \mathrm{s} n^{\circ} 7 \mathrm{vol} .4$, septembre 88 étude tout à fait intéressante de Krontiris [24] semblait indiquer une distribution différente de ces allèles $c$ Ha-ras dans des cancers de diverses origines par comparaison à des populations non affectées. Les auteurs suggéraient que la présence, avec une grande fréquence, d'allèles rares chez les cancéreux était liée à une sensibilité accrue des individus à développer un cancer. Ces conclusions ayant une implication clinique majeure, il nous a semblé intéressant de vérifier ces résultats sur une série homogène importante de cancers du col de l'utérus traités à l'Institut Gustave-Roussy.

Nous avons choisi une population témoin de 69 donneurs de sang sains chez lesquels nous avons prélevé les lymphocytes. Après la digestion par $M s p I$ de l'ADN génomique provenant de 105 cancers du col et de ces lymphocytes, nous avons révélé par hybridation avec la sonde $\mathrm{c}$-Ha-rasVTR un ou deux fragments d'ADN chez les individus homozygotes et hétérozygotes respectivement. La taille et la distribution des différents allèles décelés dans la population normale et dans les cancers du col ont été analysées. On s'aperçoit que les fréquences combinées des allèles
Tableau II

RISQUE RELATIF DE RECHUTE ESTIMÉ EN FONCTION DE L'EXPRESSION DU GĖNE C-MYC

\begin{tabular}{|c|c|c|c|}
\hline \multirow{2}{*}{$\begin{array}{l}\begin{array}{l}\text { Facteurs pris } \\
\text { en compte }\end{array} \\
\text { Surexpression } \\
\text { de } c \text {-myc }\end{array}$} & \multirow[t]{2}{*}{$\begin{array}{l}\text { Nombre de } \\
\text { malades }\end{array}$} & \multicolumn{2}{|c|}{$\begin{array}{c}\text { Risque relatif de rechute } \\
R R 1^{a}(p) \\
R R 2^{b}(p)\end{array}$} \\
\hline & & & \\
\hline $\begin{array}{l}\text { non } \\
\text { oui }\end{array}$ & $\begin{array}{l}47 \\
25\end{array}$ & $\begin{array}{l}1^{c} \\
7,6(0,0001)\end{array}$ & $\begin{array}{l}1 \\
8,0(0,001)\end{array}$ \\
\hline \multicolumn{4}{|l|}{$\begin{array}{l}\text { Envahissement } \\
\text { ganglionnaire }\end{array}$} \\
\hline $\begin{array}{l}\text { non } \\
\text { oui } \\
\text { inconnu }\end{array}$ & $\begin{array}{r}42 \\
24 \\
6\end{array}$ & $\begin{array}{l}1^{c} \\
4,2(0,01) \\
3,6\end{array}$ & $\begin{array}{l}1 \\
3,6(0,01) \\
-\end{array}$ \\
\hline \multicolumn{4}{|l|}{ Age (an) } \\
\hline $\begin{array}{l}25-44 \\
50-89\end{array}$ & $\begin{array}{l}44 \\
28\end{array}$ & $\begin{array}{l}1^{\mathrm{c}} \\
0,4 \text { (NS) }\end{array}$ & $\overline{-}$ \\
\hline \multicolumn{4}{|l|}{ Stade (UICC) } \\
\hline II & $\begin{array}{l}40 \\
32\end{array}$ & $\begin{array}{l}1^{\mathrm{c}} \\
1,1 \text { (NS) }\end{array}$ & $\overline{-}$ \\
\hline \multicolumn{4}{|l|}{$\begin{array}{l}\text { Origine } \\
\text { géographique }\end{array}$} \\
\hline $\begin{array}{l}\text { Européenne } \\
\text { Africaine }\end{array}$ & $\begin{array}{l}40 \\
32\end{array}$ & $\begin{array}{l}1^{c} \\
4,3(0,01)\end{array}$ & - \\
\hline
\end{tabular}

(a) risque relatif de rechute associé à chaque facteur, analyse unifactorielle. (b) : risque relatif de rechute estimé, analyse multifactorielle. (c) catégorie de référence. 


\section{RÉFÉRENCES}

15. Beaudenon S, Kremsdorf D, Obalek S, et al. Plurality of genital human papillomaviruses : characterization of two new types with distinct biological properties. Virology 1987 . 161 : 374-84.

16. Pratili MA, Le Doussal V, Harvey $P$, et al. Recherche de papillomavirus humains dans des cellules épithéliales du col utérin: fréquence des types 16 et 18. J Obstet Biol Reprod $1986 ; 15: 45.50$.

17. Campion MJ, McCance DJ, Cuzick J Singer A. Progressive potential of mild cervical atypia : prospective cytological colposcopic, and virological study. Lancet 1986; ii 237-40.

18. Barrasso R, De Brux J, Croissant O, Orth G. High prevalence of papillomavirusassociated penile intraepithelial neoplasia in sexual partners of women with cervical intraepithelial neoplasia. $N$ Engl J Med 1987 ; 317 : 916-23.

19. Dürst M, Croce CM, Gissmann L SchwarzE, Huebner K. Papillomavirus sequences integrate near cellular oncogenes in some cervical carcinomas. Proc Natl Acad Sci USA 1987 ; 84 : 1070-4.

20. Alitalo K, Schwab M. Oncogene amplification in tumor cells. Adv Cancer Res 1986 $47: 235-81$.

21. Bishop JM. The molecular genetics of cancer. Science 1987 ; 235 : 305-11.

22. Barbacid M. ras genes. Ann Rev Biochem $1987 ; 56$ : 779-827.

23. Riou G, Barrois M, Lê MG, George M, Le Doussal V, Haie C. $C$-myc proto-oncogene expression and prognosis in early carcinoma of the uterine cervix. Lancet 1987; i : 761-3.

24. Krontiris TG, DiMartino NA, Colb M, Parkinson DR. Unique allelic restriction fragments of the human Ha-ras locus in leukocyte and tumour DNAs of cancer patients. Nature $1985 ; 313$ : 369-74.

25. Riou G, Barrois M, Sheng ZM, Duvillard P, Lhomme C. Somatic deletions and mutations of $c$-Ha-ras gene in human cervical cancers. Oncogene 1988 (sous presse).

26. Klein G. The approaching era of the tumor suppressor genes. Science $1987 ; 238$ : 1539-45.

27. Knudson AG. A two-mutation model for human cancer. Adv Viral Oncol 1987; 7 : $1 \cdot 16$.

28. Bos JL, Fearon ER, Hamilton SR, et al. Prevalence of ras gene mutations in human colorectal cancers. Nature 1987 ; 327 : 293-7.

29. Forrester K, Almoguera C, Han K. Grizzle WE, Perucho M. Detection of high incidence of $K$-ras oncogenes during human colon tumorigenesis. Nature 1987; 327 : 298-

\begin{tabular}{|c|c|c|}
\hline \multicolumn{3}{|c|}{$\begin{array}{l}\text { Tableau III } \\
\text { RATIONS DU GĖNE C-HA-RAS-1 } \\
\text { S CANCERS DU COL DE L'UTÉRUS }\end{array}$} \\
\hline \multirow{2}{*}{$\begin{array}{l}\text { Stades } \\
\text { cliniques }\end{array}$} & \multicolumn{2}{|c|}{$\begin{array}{l}\text { Nombre de tumeurs ayant une altération } \\
\text { du gène/Nombre total de tumeurs analysées }\end{array}$} \\
\hline & $\begin{array}{c}\text { Perte d'allèle } \\
\text { (\%) }\end{array}$ & $\begin{array}{c}\text { Mutation sur le codon } 12 \\
(\%)\end{array}$ \\
\hline $1+11$ & $\begin{array}{l}7 / 21 \\
(33 \%)\end{array}$ & $\begin{array}{l}1 / 47 \\
(2 \%)\end{array}$ \\
\hline III + IV & $\begin{array}{c}4 / 13 \\
(31 \%)\end{array}$ & $\begin{array}{c}7 / 29 \\
(24 \%)\end{array} \quad(p<0,01)$ \\
\hline Total & $\begin{array}{l}11 / 34 \\
(32 \%)\end{array}$ & $\begin{array}{l}8 / 76 \\
(11 \%)\end{array}$ \\
\hline
\end{tabular}

communs et des allèles rares ne sont pas significativement différentes dans ces deux populations. En conséquence, la présence d'allèles variants sur le locus c-Ha-ras-1 ne semble pas constituer un marqueur effectif de la prédisposition génétique des individus à développer un cancer du col de l'utérus [25].

- Perte d'allèle c-Ha-ras dans les cancers. Nous avons également analysé l'ADN génomique de 53 cancers du col et des lymphocytes de ces malades dans les conditions décrites dans le paragraphe précédent. Nous avons montré la perte d'un allèle $c$ -
Ha-ras dans $32 \%$ des tumeurs provenant des sujets hétérozygotes [25] Cette perte d'hétérozygotie ne semble cependant pas associée à l'agressivité des cancers (Tableau III). Nous donnons une illustration de ce résultat dans la figure 5. La perte d'hétérozygotie de certains gènes a été largement décrite dans les rétinoblastomes et les néphroblastomes (tumeurs de Wilms), deux tumeurs de l'enfant apparaissant à la fois d'une manière héréditaire et sporadique [26]. La délétion de séquences de gènes normaux démasquerait certaines mutations récessives qui contribueraient

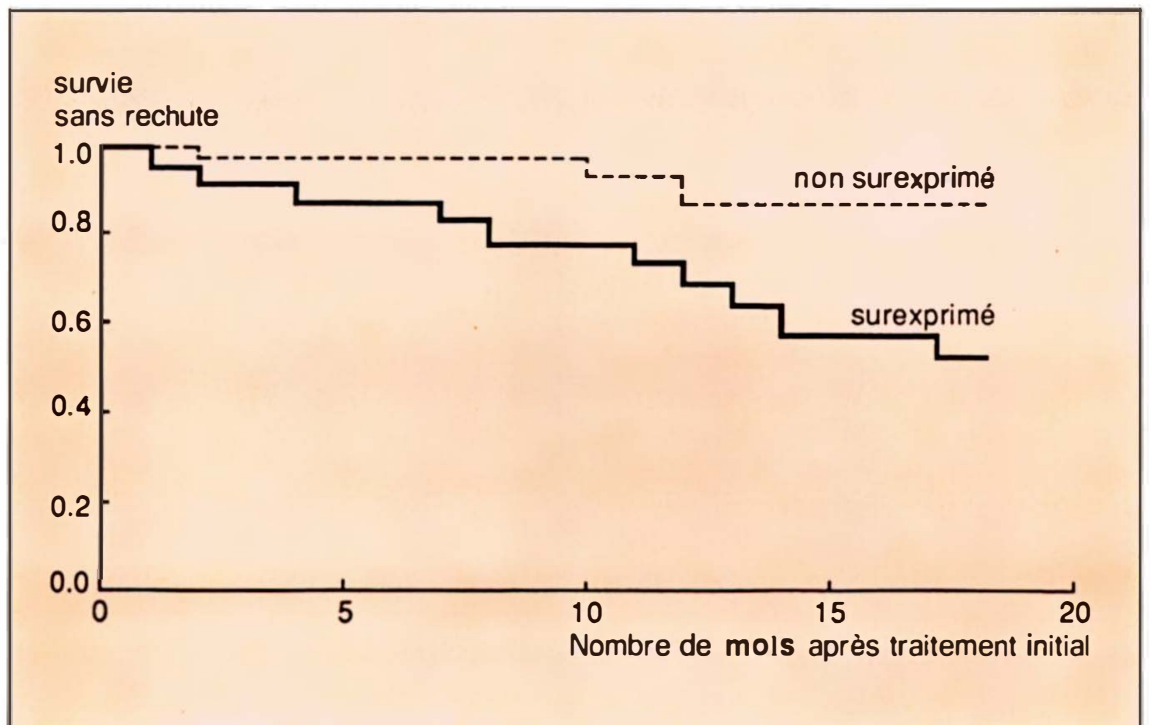

Figure 6. Courbes de survie sans rechute des malades dont les tumeurs présentent un niveau normal (-----) ou élevé (-) de transcrits c-myc. 
au développement de ces deux cancers. Cette hypothèse formulée par Knudson [27] a été ensuite étendue à d'autres tumeurs dans lesquelles des gènes situés sur le bras court du chromosome 11 ou sur d'autres chromosomes sont délétés, indiquant que de telles altérations sont importantes dans l'initiation et le développement de ces cancers.

- Mutation sur le codon 12 de $\mathrm{c}-\mathrm{Ha}$ ras dans les cancers des stades évolués. La digestion de l'ADN génomique par MspI peut également révéler les mutations survenant sur le codon $12 \mathrm{du}$ gène $c$-Ha-ras. Le gène muté supprime un site de restriction de $M s p I$ et engendre un fragment d'ADN de 411 paires de base alors que le gène non muté fournit 2 fragments d'ADN de 355 et 56 paires de base. Sur les 78 tumeurs étudiées, nous avons décelé huit fois une mutation affectant les deux allèles (comme dans la cellule EJ)* ou un seul allèle (Tableau III). Nous illustrons également ces résultats dans la figure 5. Les mutations sont plus fréquemment observées dans les cancers des stades évolués (III et IV). Ces résultats suggèrent que les mutations sont associées à l'agressivité des cancers du col. Il est tout à fait possible que ces mutations, déjà présentes dans les cancers de stade précoce, soient responsables de leur rapide évolution. De nouvelles techniques d'amplification et d'hybridation à l'aide de sondes polynucléotidiques permettent d'analyser les mutations en positions 12,13 et 61 des gènes $c$ $\mathrm{Ha}$-ras, $c$-Ki-ras et $\mathrm{N}$-ras. Il est permis d'envisager que de telles mutations soient également décelées dans les cancers du col de l'utérus. C'est en utilisant de telles techniques que deux équipes de chercheurs [28, 29] viennent de montrer la présence de mutations du gène $c$-Ki-ras dans plus de $30 \%$ des cancers du côlon. Ces mutations sont également présentes dans les lésions précancéreuses. Les auteurs suggèrent que ce sont ces lésions qui pourraient se transformer ultérieurement en cancers. Les mutations que nous avons décelées dans les cancers invasifs du col pouvaient

* Cellules EJ : cellules cancéreuses humaines établies en lignée à partir d'un cancer de la vessie. Le gène c-Ha-ras est affecté d'une mutation sur le codon 12, conférant à ce gène des propriétés oncogènes [22]. être déjà présentes dans les lésions précancéreuses et il serait tout à fait souhaitable de les analyser dans ces lésions et les carcinomes in situ. La présence de papillomavirus oncogènes et de mutations dans de telles lésions permettrait de déceler les sujets à risque de développer un cancer invasif. Il est également intéressant de noter que les cancers du col dans lesquels on a observé la mutation du gène $c$-Ha-ras présentaient également un gène $c-m y c$ amplifié et/ou surexprimé. Ces résultats semblent démontrer que ces deux protooncogènes pourraient coopérer pour susciter une plus grande agressivité des cellules tumorales du col de l'utérus.

\section{Conclusions}

Les événements génétiques qui aboutissent à la transformation d'une cellule normale en cellule cancéreuse semblent multiples. Nous avons vu que les séquences de papillomavirus sont intégrées dans le génome cellulaire de plus de $90 \%$ des cancers invasifs et qu'ils sont exprimés dans plus de $80 \%$ des cas. Ces virus sont vraisemblablement impliqués dans l'initiation des cancers du col et peut-être dans le maintien de l'état malin et dans l'activation d'oncogènes. L'étude in vitro de modèles cellulaires de cancer du col permettra peut-être de mettre en évidence le rôle de ces virus dans ce processus d'activation. Nous avons également vu que les proto-oncogènes $c-m y c$ et $c$ Ha-ras étaient associés à la progression tumorale. Leur activation décelée dans les cancers de stade précoce semble avoir une valeur pronostique intéressante. Il est de plus probable que d'autres proto-oncogènes impliqués dans ce cancer seront découverts. Les cliniciens auront alors à leur disposition de nouveaux marqueurs qui permettront d'envisager une action thérapeutique plus précoce et plus efficace.

Nous concluerons sur le problème majeur qui va se poser : comment le transfert de la technologie sophistiquée de biologie moléculaire dans les laboratoires de biologie de routine va-t-elle se faire? L'une des issues possibles est l'analyse de la protéine dans les tissus tumoraux par hybridation in situ à l'aide d'anticorps monoclonaux

\section{Summary}

Although in developped countries cervical cancer seems to regress, its frequency is always elevated and this cancer raises an acute health problem. Actually, one of major problems is the lack of assessed prognostic factors which do not predict the evolution of early cancers. It is quite well admitted that human papillomaviruses are important etiological factors and that their detection in precancerous lesions is a risk factor for malignant transformation. If these viruses seem to play a role in initiation step of cervical cancer, other factors are necessary to the malignant transformation. Among those, certain proto-oncogenes (c-myc and $c-\mathrm{Ha}$ ras) seem to be involved in the development and progression of cancer. Detection of these gene alterations (mutation, deletion, amplification) and of their overexpression in precancerous lesions and in early invasive cancers could be useful in the establishment of prognosis. A better surveillance of these high risk patients and a more appropriate treatment could be applied.

\section{Remerciements}

Nous remercions M. Barrois, M. Gabillot (laboratoire de pharmacologie clinique et moléculaire), les médecins, infirmières et secrétaires de l'Institut Gustave-Roussy qui nous ont permis de faire cette étude (service de chirurgie, Dr G. Michel; service de gynécologie, Dr C. Lhomme ; service de curiethérapie, Dr A. Gerbaulet ; laboratoire d'histopathologie B, Dr M. Prade). Nous remercions particulièrement le Dr P. Duvillard (laboratoire d'histopathologie B) qui nous a fourni les clichés des figures 1,2 et 3. 